\title{
On the Study of Analogue Network Coding for Multi-Pair, Bidirectional Relay Channels
}

\author{
Chee Yen Leow, Student Member, IEEE, Zhiguo Ding, Member, IEEE, Kin K. Leung, Fellow, IEEE, \\ and Dennis L. Goeckel, Senior Member, IEEE
}

\begin{abstract}
We consider a scenario where multiple pairs of users exchange information within pair, with the help of a dedicated multi-antenna relay. The protocol integrates the idea of analogue network coding in mixing two data streams originating from the same user pair, together with the spatial multiplexing of the data streams originating from different user pairs. The key feature of the protocol is that it enables both the relay and the users to participate in interference cancellation. We propose several beamforming schemes for the multi-antenna relay and evaluate the performance using information theoretical metrics such as ergodic capacity, outage probability and diversity and multiplexing tradeoff. Analytical and simulation results justify that the ergodic capacity, outage probability and diversity and multiplexing tradeoff of the proposed beamforming schemes outperform comparable schemes.
\end{abstract}

Index Terms-Network coding, bidirectional relaying, spatial multiplexing, multi-pair interference, self-interference.

\section{INTRODUCTION}

$\mathbf{N}$ ETWORK coding [1] is a powerful technique which allows intermediate nodes to mix the signals from multiple links in order to enhance the network throughput. The application of network coding in wireless networks has received growing interest in recent years. Based on the idea of network coding, bidirectional relaying in wireless networks is able to enhance the network throughput by reducing the channel resources used in the information exchange between

Manuscript received June 10, 2010; revised August 23, 2010; accepted October 12, 2010. The associate editor coordinating the review of this paper and approving it for publication was H. Ochiai.

This research was sponsored by the US Army Research Laboratory and the UK Ministry of Defence and was accomplished under Agreement Number W911NF-06-3-0001. The views and conclusions contained in this document are those of the authors and should not be interpreted as representing the official policies, either expressed or implied, of the US Army Research Laboratory, the U.S. Government, the UK Ministry of Defense, or the UK Government. The US and UK Governments are authorized to reproduce and distribute reprints for Government purposes notwithstanding any copyright notation hereon. The authors would like to thank Prof. Don Towsley from University of Massachusetts and Ananthram Swami from the US Army Research Laboratory for their valuable comments and suggestions.

C. Y. Leow is with the Dept. of Electrical \& Electronic Engineering, Imperial College London, UK, and the Faculty of Electrical Engineering, Universiti Teknologi Malaysia (e-mail: bruceleow@imperial.ac.uk).

Z. Ding is with the School of Electrical, Electronic \& Computer Engineering, Newcastle University, UK (e-mail: z.ding@newcastle.ac.uk).

K. K. Leung is with the Dept. of Electrical \& Electronic Engineering, Imperial College London, UK (e-mail: kin.leung@imperial.ac.uk).

D. L. Goeckel is with the Dept. of Electrical \& Computer Engineering, University of Massachusetts, Amherst, US (e-mail: goeckel@ecs.umass.edu).

Z. Ding was supported by the UK EPSRC under grant number EP/F062079/2.

Digital Object Identifier 10.1109/TWC.2010.120610.101017 nodes. Bidirectional relaying schemes such as strategies summarised in [2] based on decode-and-forward (DF) relaying, analogue network coding [3] based on amplify-and-forward (AF) relaying, physical network coding [4] based on estimateand-forward (EF) relaying, etc are able to complete the two way information passing in only two phases. Since the total channel use is halved, the number of independent data streams that can be transmitted or received simultaneously per channel use in the network is doubled, as compared to the time division protocol which requires 4 orthogonal channel uses.

Attracted by the benefits of multi-antenna in enhancing the system capacity and reliability, bidirectional relaying has been generalised to the multi-antenna case. [5] generalises the DF based bidirectional relaying to multi-antenna setting using classical multiple access capacity region and devises an optimal broadcast strategy based on point-to-point multiple-input multiple-output (MIMO). On the other hand, [6] proposes an $\mathrm{AF}$ based protocol which uses zero-forcing beamformer to eliminate the co-channel interference between users. However, no network coding is used to mix the data streams from two users. [7] and [8] consider the sum rate optimising AF based beamforming design, for the case where only the relay is equipped with multi-antenna. Based on analogue network coding, the beamforming schemes proposed in [7] and [8] are able to deliver sum rate improvement if compared with [6].

The extension of the bidirectional relaying to multiple user pairs introduces the problem of multi-user interference. [9] proposes a scheme for CDMA system where each pair of users shares a common spreading code as a mean to reduce the multi-user interference. The proposed demodulate-andforward based scheme in [9] uses multi-user receiver which requires high computational complexity at the relay. The suboptimal AF based scheme proposed in [9] suffers from poor BER performance when the number of users is low, due to noise domination. [10] proposes a scheme using DF based relay with multiple antennas for narrow band system, where XOR precoding is used at the relay to encode the messages from the same user pair while block-diagonalisation [11] is used to mitigate the interference caused by multi-pair. Since DF based scheme requires higher complexity for decoding/encoding at the relay as compared to the AF based scheme, [12] and [13] consider the multi-pair scenario with an AF based multiantenna relay. Similar to [10], the schemes in [12] and [13] use the idea of block-diagonalisation [11] to eliminate the multi-pair interference and to forward the mixture containing the desired message and the self-interference to the each 
user. Each user then uses the knowledge of the previously transmitted message to subtract the self-interference from the mixture to decode the new message. The schemes in [12] and [13] have shown that a higher sum rate can be achieved in comparison with the conventional multi-user zeroforcing scheme. Nevertheless, the potential benefit of blockdiagonalisation in the scenario where the relay does not have enough degrees-of-freedom to spatially separate and/or decode each independent message is not covered in [10], [12], [13]. Furthermore, the spatial diversity gain offered by blockdiagonalisation in the multi-pair scenario has not been studied.

In this paper, we consider a scenario where multiple pairs of users exchange information within pair, with the help of a dedicated AF based, multi-antenna relay. The transmission protocol employed in this paper utilises the principal concept of network coding in mixing two data streams originating from the same user pair, coupled with the spatial multiplexing of the data streams originating from different user pairs. The key feature of the protocol is that it enables both the relay and the users to participate in interference cancellation: the relay eliminates co-channel interference due to multi-pair while each user eliminates the self-interference. We propose several low complexity beamforming schemes based on the idea of block-diagonalisation, and evaluate their performance using information theoretical metrics, such as the ergodic capacity, outage probability and the diversity and multiplexing tradeoff. Two cases have been considered. First, the case where the number of antennas at the relay is less than the total singleantenna users. Second, the case where the number of antennas at the relay is at least the total single-antenna users. In the first case, simulation results show that our proposed beamforming scheme is able to deliver significant ergodic capacity improvement and higher multiplexing gain if compared with existing schemes based on time sharing between pairs. Upper and lower bounds on ergodic capacity achieved by the proposed scheme are derived to quantify the performance. In the second case, we propose two beamforming schemes and show that appropriate selection or coherent combining of null-space vectors is able to achieve all the available diversity gain offered by block-diagonalisation. The proposed beamforming with coherent combining of null-space vectors achieves the highest ergodic capacity and the lowest outage probability among all comparable schemes, while the proposed beamforming with null-space vector selection performs close to the former. The proposed beamforming schemes deliver higher diversity gain as compared with existing zero-forcing scheme [6] while several comparable schemes based on block-diagonalisation [12], [13] fail to do so due to non-coherent combining of the diversity streams. The proposed schemes also offer better rate and reliable tradeoff as compared to the zero-forcing scheme [6] and the comparable schemes based on time sharing between pairs [7]. Analytical results on outage probability and the diversity and multiplexing tradeoff are derived to quantify the achievable performance.

This paper is organised as following. The system model and protocol are described in section II, while the proposed beamforming schemes are discussed in section III. The analytical results on the ergodic capacity are presented in section IV, followed by the analytical results on the outage probability and
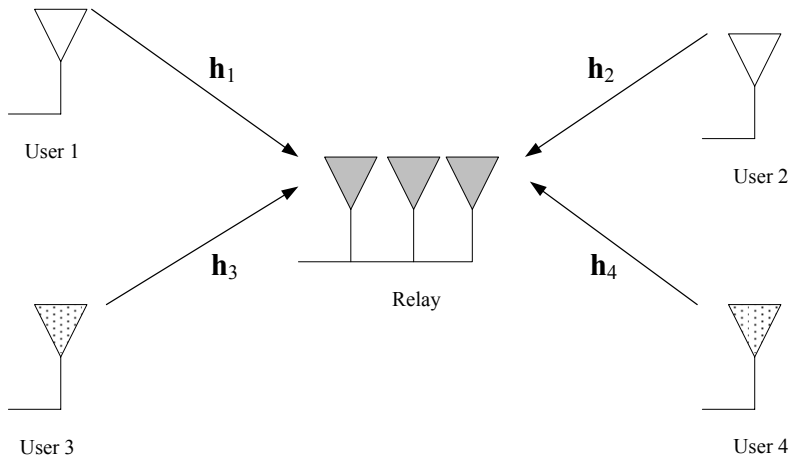

Fig. 1. Scenario when 2 pairs of single-antenna users exchanging information with the help of a multi-antenna relay.

the diversity and multiplexing tradeoff in section V. In section VI, the numerical results on the ergodic capacity and the outage probability are discussed. The last section concludes the paper. Part of the contents of this paper is presented in [14].

\section{System Model And Protocol Description}

We consider a scenario where there are $M$ pairs of singleantenna users who wish to exchange information with their partners, with the help of a dedicated AF based relay equipped with $N$ antennas. An example of the scenario where $M=2$ and $N=3$ is shown in fig. 1 .

We assume the symmetric case, where all nodes are subjected to unit average power constraint and have the same channel statistics. All channels undergo i.i.d. quasi-static Rayleigh fading and channel reciprocity is assumed. The receiver is corrupted by circularly symmetric additive white Gaussian noise with distribution $\mathcal{C} \mathcal{N} \sim\left(0, \sigma^{2}\right)$. Half duplex constraint is assumed throughout the paper and it is realised using time division duplexing. Every user knows his and his partner's effective user-to-relay channel state information (CSI) while the relay has the CSI of all user-to-relay links.

We use $(i, j)$ to represent the pair of user $i$ and user $j$ who exchange information with each other, such that the $m$ th user pair is denoted as $(2 m-1,2 m)$. The channel from user $i$ to the relay is $\mathbf{h}_{i} \in \mathbb{C}^{N \times 1}$, the message transmitted from user $i$ is $x_{i} \in \mathbb{C}$, the combined channels of user pair $(i, j)$ is $\mathbf{H}_{i, j}=\left[\begin{array}{ll}\mathbf{h}_{i} & \mathbf{h}_{j}\end{array}\right] \in \mathbb{C}^{N \times 2}$ and the message vector of user pair $(i, j)$ is $\mathbf{x}_{i, j}=\left[\begin{array}{ll}x_{i} & x_{j}\end{array}\right]^{T} \in \mathbb{C}^{2 \times 1}$ where [.] $]^{T}$ denotes the transpose operation. The noise observed by the relay and user $i$ is $\mathbf{n} \in \mathbb{C}^{N \times 1}$ and $n_{i} \in \mathbb{C}$ respectively. We define the multi-pair interference ${ }^{1}$ channel seen by user pair $(i, j)$ as $\tilde{\mathbf{H}}_{i, j} \in \mathbb{C}^{N \times 2(M-1)}$ by stacking all user channels other than $\mathbf{H}_{i, j}$. Similarly, we define the message vector conveyed through $\tilde{\mathbf{H}}_{i, j}$ as $\tilde{\mathbf{x}}_{i, j} \in \mathbb{C}^{2(M-1) \times 1}$ by stacking all messages other than $\mathbf{x}_{i, j}$.

The employed protocol combines the application of analogue network coding within pair and spatial multiplexing between pairs, thus we name it as network coding with spatial multiplexing (NC-SM) protocol. The NC-SM protocol can be

\footnotetext{
${ }^{1}$ The multi-pair interference is different from the multi-user interference defined in the literature. The multi-user interference is the interference observed by each user, not by each pair.
} 
described in two time slots. In the first time slot, $M$ pairs of users transmit simultaneously in the same channel with unit power. The relay observes a mixture of all messages from the users, which can be expressed as

$$
\mathbf{r}=\sum_{m=1}^{M} \mathbf{H}_{i, j} \mathbf{x}_{i, j}+\mathbf{n}
$$

where $i=2 m-1, j=2 m$. In the second time slot, the AF based relay broadcasts the linearly processed observation, i.e. Fr, where $\mathbf{F} \in \mathbb{C}^{N \times N}$ is the beamforming matrix at the relay. The signal received by user $i$ can be expressed as

$$
y_{i}=\mathbf{h}_{i}^{T} \mathbf{F} \mathbf{H}_{i, j} \mathbf{x}_{i, j}+\mathbf{h}_{i}^{T} \mathbf{F} \tilde{\mathbf{H}}_{i, j} \tilde{\mathbf{x}}_{i, j}+\mathbf{h}_{i}^{T} \mathbf{F n}+n_{i} .
$$

The first term on the RHS of the equation contains the mixture of messages from user pair $(i, j)$, the second term contains the multi-pair interference while the last two terms contain the relay propagated noise and the receiver noise of user $i$. The unique feature of the NC-SM protocol is to allow both the relay and the users to participate in the interference cancellation. The relay eliminates the multi-pair interference, $\mathbf{h}_{i}^{T} \mathbf{F} \tilde{\mathbf{H}}_{i, j} \tilde{\mathbf{x}}_{i, j}$, while user $i$ remove the selfinterference, $\mathbf{h}_{i}^{T} \mathbf{F} \mathbf{h}_{i} x_{i}$. The design of beamforming matrix $\mathbf{F}$ is discussed in the following section.

\section{JOINT RECEIVE AND TRANSMIT BEAMFORMING DESIGN}

In this section, we present the low complexity beamforming design at the relay, in two cases. Case I: $N=2 M-1$, which corresponds to the case when the number of antennas at the relay is less than total number of user; and case II: $N \geq 2 M$, which corresponds to the case when the number of antennas at the relay is at least the total number of users. Subsection III-A explains case I while subsection III-B discusses case II. Note that the proposed protocol does not operate when $N<2 M-1$, due to the zero-forcing criterion, which is discussed in detail in the following subsections.

\section{A. Case I: $N=2 M-1$}

This case corresponds to the situation where conventional multi-antenna receiver or transmitter is not able to spatially support $2 M$ independent data streams, due to the limitation of the available degrees-of-freedom [15], i.e. $\min (N, 2 M)$. The scheme proposed in [10] does not work under this case due to insufficient antennas at the relay, while [12] and [13] have not explored this specific setting. It will be shown in the following paragraph that the proposed beamforming structure is able to support $2 M$ independent data streams (from $2 M$ users) simultaneously, given only $N=2 M-1$, by aligning the data streams of each user pair to occupy only 1 spatial dimension. As a result, a higher multiplexing gain can be achieved.

The proposed beamforming matrix $\mathbf{F}$ consists of the receive beamforming matrix $\mathbf{W}_{R}$ and transmit beamforming matrix $\mathbf{W}_{T}$, which are directly cascaded as follows,

$$
\mathbf{F}=\mathbf{W}_{T} \mathbf{A} \mathbf{W}_{R},
$$

where the receive beamforming matrix $\mathbf{W}_{R} \in \mathbb{C}^{M \times N}$ and the transmit beamforming matrix $\mathbf{W}_{T} \in \mathbb{C}^{N \times M}$ while the diagonal matrix $\mathbf{A} \in \mathbb{R}^{M \times M}$ is the power allocation matrix. Due to channel reciprocity, $\mathbf{W}_{T}=\mathbf{W}_{R}^{T}$. This allows us to concentrate on the design of the transmit beamforming matrix. For simplicity, we omit the subscript and let $\mathbf{W}_{T}=\mathbf{W}$. Represent $\mathbf{W}=\left[\begin{array}{lll}\mathbf{w}_{1,2} & \ldots & \mathbf{w}_{2 M-1,2 M}\end{array}\right]$ where $\mathbf{w}_{i, j} \in$ $\mathbb{C}^{N \times 1}$ is the transmit beamforming vector for user pair $(i, j)$, $\mathbf{A}=\operatorname{diag}\left(\alpha_{1,2} \quad \ldots \quad \alpha_{2 M-1,2 M}\right)$, and $\mathbf{F}_{i, j} \in \mathbb{C}^{N \times N}=$ $\mathbf{w}_{i, j} \mathbf{w}_{i, j}^{T}$ as the effective beamforming matrix for pair $(i, j)$. We can rewrite (3) as $\mathbf{F}=\sum_{m=1}^{M} \alpha_{i, j} \mathbf{F}_{i, j}$ where $i=$ $2 m-1, j=2 m$. The design objective of $\mathbf{w}_{i, j}$ is to ensure that each user pair is free from the multi-pair interference. In other words, the zero-forcing criterion $\tilde{\mathbf{H}}_{i, j}^{T} \mathbf{w}_{i, j}=\mathbf{0}$ has to be satisfied for all pair $(i, j)$, where $\mathbf{0}$ is a column vector of all zeros. This criterion coincides with the blockdiagonalisation $^{2}$ for the MIMO broadcast channels in [11]. To satisfy this criterion, we choose $\mathbf{w}_{i, j}$ to lie in the null-space of the multi-pair interference channel, i.e. $\mathbf{w}_{i, j}=\operatorname{null}\left(\tilde{\mathbf{H}}_{i, j}^{T}\right)$, which exists as a non-zero vector when $N=2 M-1$. Note that $\operatorname{rank}\left(\mathbf{H}_{i, j}^{T} \mathbf{w}_{i, j}\right)=1$, i.e. each user pair only occupies one spatial dimension. This enables the relay to spatially multiplex $2 M$ independent streams by using only $N=2 M-1$ antennas.

The transmission from the relay is subjected to unit average power constraint. The power constraint can be expressed as

$$
\sum_{m=1}^{M} \alpha_{i, j}^{2}\left(\left\|\mathbf{F}_{i, j} \mathbf{H}_{i, j}\right\|_{F}^{2}+\sigma^{2}\left\|\mathbf{F}_{i, j}\right\|_{F}^{2}\right) \leq 1,
$$

where $i=2 m-1, j=2 m$, and \|\|$_{F}$ denotes the Frobenius norm. Note that the expected value, $E\left[\mathbf{n n}^{H}\right]=$ I. Since we are interested in the high SNR performance, i.e. diversity and multiplexing gains, equal power allocation across $M$ data streams from $M$ user pairs is sufficient. Although optimal power allocation among user pairs is able to further improve the sum rate performance, it improves neither the diversity gain nor the multiplexing gain. Using equal power allocation, the equation above is satisfied in equality by choosing $\alpha_{i, j}=$ $\frac{1}{\sqrt{M}} \frac{1}{\sqrt{\left\|\mathbf{F}_{i, j} \mathbf{H}_{i, j}\right\|_{F}^{2}+\sigma^{2}}}$. Note that $\left\|\mathbf{F}_{i, j}\right\|_{F}^{2}=\left\|\mathbf{w}_{i, j} \mathbf{w}_{i, j}^{T}\right\|_{F}^{2}=$ 1. The signal received by user $i$ can be expressed as

$$
y_{i}=\alpha_{i, j} \mathbf{h}_{i}^{T} \mathbf{F}_{i, j}\left(\mathbf{h}_{i} x_{i}+\mathbf{h}_{j} x_{j}+\mathbf{n}\right)+n_{i},
$$

while the signal received by user $j$ is

$$
y_{j}=\alpha_{i, j} \mathbf{h}_{j}^{T} \mathbf{F}_{i, j}\left(\mathbf{h}_{i} x_{i}+\mathbf{h}_{j} x_{j}+\mathbf{n}\right)+n_{j} .
$$

Note that for all $p \neq i$ and $q \neq j$, we have $\mathbf{h}_{i}^{T} \mathbf{F}_{p, q} \mathbf{h}_{i}=0$, $\mathbf{h}_{i}^{T} \mathbf{F}_{p, q} \mathbf{h}_{j}=0, \mathbf{h}_{j}^{T} \mathbf{F}_{p, q} \mathbf{h}_{i}=0$ and $\mathbf{h}_{j}^{T} \mathbf{F}_{p, q} \mathbf{h}_{j}=0$. Since user $i$ has the knowledge of $x_{i}$, and the knowledge of the effective channels, $\mathbf{h}_{i}^{T} \mathbf{F}_{i, j} \mathbf{h}_{i}$ and $\mathbf{h}_{i}^{T} \mathbf{F}_{i, j} \mathbf{h}_{j}$, he can decode the desired message, $x_{j}$, by subtracting the self-interference, $\alpha_{i, j} \mathbf{h}_{i}^{T} \mathbf{F}_{i, j} \mathbf{h}_{i} x_{i}$, from the received mixture. Similar strategy is used by user $j$ to decode the desired message, $x_{i}$. Notice that the effective channels are scalars. The effective scalar channels carrying self-interference, $\mathbf{h}_{i}^{T} \mathbf{F}_{i, j} \mathbf{h}_{i}$ and $\mathbf{h}_{j}^{T} \mathbf{F}_{i, j} \mathbf{h}_{j}$, can be fed back from the relay to user $i$ and $j$ respectively

\footnotetext{
${ }^{2}$ Each user pair in the multi-pair scenario considered here is analogous to each multi-antenna user in the MIMO broadcast channels considered in [11] However, different from the MIMO broadcast channels, the users in each pair are not able to cooperate with each other, i.e. linear postprocessing within user pair is not possible. Hence, the block-diagonalisation proposed in [11] cannot be directly applied in the multi-pair scenario.
} 
using orthogonal feedback channels, while the effective scalar channel carrying desired message, $\mathbf{h}_{i}^{T} \mathbf{F}_{i, j} \mathbf{h}_{j}$, can be fed back from the relay to user pair $(i, j)$ simultaneously using a common feedback channel, with low overhead. Note that $\mathbf{h}_{i}^{T} \mathbf{F}_{i, j} \mathbf{h}_{j}=\mathbf{h}_{j}^{T} \mathbf{F}_{i, j} \mathbf{h}_{i}$. User pair $(i, j)$ do not need to know the exact channel vectors, $\mathbf{h}_{i}$ and $\mathbf{h}_{j}$.

Assuming Gaussian channel coding, the mutual information of user $i$ can be described as

$$
\mathcal{I}_{i}=\frac{1}{2} \log _{2}\left(1+\frac{\alpha_{i, j}^{2}\left|\mathbf{h}_{i}^{T} \mathbf{F}_{i, j} \mathbf{h}_{j}\right|^{2}}{\sigma^{2}\left(\alpha_{i, j}^{2}\left\|\mathbf{h}_{i}^{T} \mathbf{F}_{i, j}\right\|^{2}+1\right)}\right) .
$$

where |.| and ||.|| denote the absolute value and Euclidean norm respectively. The pre-log reflects the two time slots used to complete the information exchange. The mutual information of user $j, \mathcal{I}_{j}$ can be obtained by interchanging $\mathbf{h}_{i}$ and $\mathbf{h}_{j}$ in (7). Define a floating point operation as one complex multiplication or addition. We use the number of floating point operations (flops) to measure the computational complexity. It can be easily shown that the computational complexity of the proposed scheme is at most $\mathcal{O}\left(M N^{3}\right)$ flops. Generally, this beamforming scheme performs well when both channels $\mathbf{h}_{i}$ and $\mathbf{h}_{j}$ have reasonably good quality ${ }^{3}$.

\section{B. Case II: $N \geq 2 M$}

Similar to the previous case, the design objective of the beamforming matrix $\mathbf{F}$ is to ensure that the multi-pair interference is nullified. However, different from the previous case, the dimension of $\operatorname{null}\left(\tilde{\mathbf{H}}_{i, j}^{T}\right)$ is greater than 1, indicating that the null-space consists of multiple vectors. The transmit beamforming matrix for pair $(i, j)$ is now $\mathbf{W}_{i, j} \in \mathbb{C}^{N \times(N-2(M-1))}$, where $\mathbf{W}_{i, j}=\operatorname{null}\left(\tilde{\mathbf{H}}_{i, j}^{T}\right)$. Multiple null-space vectors are able to improve the diversity gain, by providing multiple statistically independent paths for the messages to travel through. In order to benefit from the additional diversity gain, the beamforming structure needs to be carefully designed. A trivial choice of directly cascading the receive and transmit beamforming matrices as in (3), destroys the diversity gain offered by multiple null-space vectors. This is due to the fact that the superposition of multiple diversity streams can either add up constructively or destructively at the destinations. It will be shown that appropriate selection or coherent combining of null-space vectors is important to achieve the available diversity gain offered by block-diagonalisation based beamforming in comparison with the zero-forcing scheme [6]. The block-diagonalisation with singular value decomposition (BD-SVD) [13] and pair-aware matched filter (PA-MF) [12] fail to achieve the available diversity gain because the diversity streams are not coherently combined. In this paper, two beamforming schemes which are able to achieve all diversity gain offered by block-diagonalisation, are proposed. One is based on null-space vector selection and the other is based on coherent combining of all null-space vectors.

\footnotetext{
${ }^{3}$ The performance degrades when either one of the channels is in deep fade. This can be solved by increasing the diversity gain, achieved by either using more antennas at the relay, as discussed in subsection III-B, or implementing user selection. When the number of users in the network is large, the relay can select the user pairs with good channel quality at a particular time instance to become active users, in order to harvest the benefit of multi-user diversity.
}

1) Null-Space Vector Selection : In this subsection, we propose a beamformer with null-space vector selection. The relay performs selection to determine the null-space vector that can deliver the best performance in maximising the sum rate of each user pair $(i, j)$. The overall beamforming structure $\mathbf{F}$ is similar to (3). Since we are interested in the high SNR performance, i.e. diversity and multiplexing gains, equal power allocation among user pairs is sufficient. Denote the $k$ th nullspace vector for pair $(i, j)$, obtained from the $k$ th column of $\mathbf{W}_{i, j}$, as $\mathbf{w}_{i, j}(k)$. We have the following null-space vector selection criterion for user pair $(i, j)$,

$$
\arg \max _{k=1, \ldots, N-2(M-1)} \mathcal{I}_{i}(k)+\mathcal{I}_{j}(k),
$$

where $\mathcal{I}_{i}(k)$ and $\mathcal{I}_{j}(k)$ are the mutual information of user $i$ and user $j$ respectively, when $\mathbf{w}_{i, j}(k)$ is used. $\mathcal{I}_{i}(k)$ can be obtained by replacing $\mathbf{F}_{i, j}=\mathbf{w}_{i, j}(k) \mathbf{w}_{i, j}^{T}(k)$ in (7) while $\mathcal{I}_{j}(k)$ can be derived similarly. The best null-space vector, denoted as $\mathbf{w}_{i, j}\left(k_{\text {best }}\right)$ is able to maximise the sum rate of user pair $(i, j)$ and is used as the receive and transmit beamforming vectors for pair $(i, j)$. The sum rate of user pair $(i, j)$ is used instead of the individual rate because the best null-space vector, $\mathbf{w}_{i, j}\left(k_{\text {best }}\right)$ affects both user $i$ and user $j$ simultaneously. In other words, each beam carries the mixture of the messages of user pair $(i, j)$. The received signal and the mutual information of user $i$ can be expressed in similar way as (5) and (7) by substituting $\mathbf{F}_{i, j}=\mathbf{w}_{i, j}\left(k_{\text {best }}\right) \mathbf{w}_{i, j}\left(k_{\text {best }}\right)^{T}$. The worst case computational complexity of this selection scheme is $\mathcal{O}\left(M N^{3}\right)$ flops, which is the same as the previous scheme. This selection scheme serves as a lower bound for the derivation of the outage probability and the diversity and multiplexing tradeoff, which are discussed in section V.

2) Coherent Combining of Null-space Vectors: In contrast to the null-space vector selection scheme, the beamformer proposed in this subsection utilises all the available null-space vectors. In order to guarantee that the superposition of multiple diversity streams at the target destination is constructive, we propose the following beamforming structure,

$$
\mathbf{F}=\sum_{m=1}^{M} \mathbf{W}_{i, j} \mathbf{B}_{i, j} \mathbf{A}_{i, j} \mathbf{P}_{\pi} \mathbf{B}_{i, j}^{T} \mathbf{W}_{i, j}^{T}
$$

where $i=2 m-1, j=2 m$. The matrix $\mathbf{W}_{i, j} \in$ $\mathbb{C}^{N \times(N-2(M-1))}$ is the transmit beamforming matrix for pair $(i, j)$, the matrix $\mathbf{B}_{i, j} \in \mathbb{C}^{(N-2(M-1)) \times 2}$ is the channel matching matrix for pair $(i, j)$, the diagonal matrix $\mathbf{A}_{i, j} \in \mathbb{R}^{2 \times 2}$ is the power allocation matrix for user pair $(i, j)$ while the matrix $\mathbf{P}_{\pi}=\left[\begin{array}{ll}0 & 1 \\ 1 & 0\end{array}\right]$ is the permutation matrix.

The channel matching matrix for pair $(i, j)$ is designed as $\mathbf{B}_{i, j}=\mathbf{W}_{i, j}^{H} \mathbf{H}_{i, j}^{*}$ where [.] ${ }^{H}$ and [.] ${ }^{*}$ denote the Hermitian transpose and complex conjugate operations respectively. We can express the effective relay to user $(i, j)$ channel as $\mathbf{H}_{i, j}^{T} \mathbf{W}_{i, j} \mathbf{B}_{i, j}=\left[\begin{array}{cc}\Phi_{i} & \Psi \\ \Psi^{*} & \Phi_{j}\end{array}\right]$, where $\Phi_{i}=$ $\sum_{k=1}^{N-2(M-1)}\left|\mathbf{h}_{i}^{T} \mathbf{w}(k)\right|^{2}, \Phi_{j}=\sum_{k=1}^{N-2(M-1)}\left|\mathbf{h}_{j}^{T} \mathbf{w}(k)\right|^{2}$ and $\Psi=\sum_{k=1}^{N-2(M-1)} \mathbf{h}_{i}^{T} \mathbf{w}(k) \mathbf{w}^{H}(k) \mathbf{h}_{j}^{*}$. The channel matching matrix $\mathbf{B}_{i, j}$ ensures that main diagonal elements $\Phi_{i}$ and $\Phi_{j}$ contain the coherently combined (at zero phase) diversity 
streams of user $i$ and user $j$ respectively, while the offdiagonal element, $\Psi$, contains the non-coherent superposition of the correlated streams for user $i$ and $j$. The permutation matrix $\mathbf{P}_{\pi}$ plays an important role to ensure that diversity gain (contributed by the coherently combined diversity streams) is preserved when the transmit beamforming matrix $\mathbf{W}_{i, j} \mathbf{B}_{i, j} \mathbf{A}_{i, j}$ and receive beamforming matrix $\mathbf{B}_{i, j}^{T} \mathbf{W}_{i, j}^{T}$ are cascaded.

The relay is subjected to unit average transmit power constraint. Since we are interested in the high SNR performance, i.e. diversity and multiplexing gains, equal power allocation among users is sufficient. Under equal power allocation, we can express $\mathbf{A}_{i, j}=\alpha_{i, j} \mathbf{I}$ and further denote $\mathbf{F}_{i, j} \in \mathbb{C}^{N \times N}=$ $\mathbf{W}_{i, j} \mathbf{B}_{i, j} \mathbf{P}_{\pi} \mathbf{B}_{i, j}^{T} \mathbf{W}_{i, j}^{T}$ such that $\mathbf{F}=\sum_{m=1}^{M} \alpha_{i, j} \mathbf{F}_{i, j}$ where $i=2 M-1$ and $j=2 M$. The power constraint can be expressed similarly as in (4). The power constraint is satisfied in equality by choosing $\alpha_{i, j}=\frac{1}{\sqrt{M}} \frac{1}{\sqrt{\left\|\mathbf{F}_{i, j} \mathbf{H}_{i, j}\right\|_{F}^{2}+\sigma^{2}|| \mathbf{F}_{i, j} \|_{F}^{2}}}$. The signal received by user $i$ can be written in the same way as in (5). User $i$ is able to decode the desired message, $x_{j}$, by subtracting the self-interference from the observation. Expand the effective channel carrying the desired message of user $i$,

$$
\alpha_{i, j} \mathbf{h}_{i}^{T} \mathbf{F}_{i, j} \mathbf{h}_{j}=\alpha_{i, j} \Phi_{i} \Phi_{j}+\alpha_{i, j}|\Psi|^{2} .
$$

The first term on the RHS of (10) contains the multiplication of the coherently combined diversity streams of user $i$, i.e. $\Phi_{i}$, and coherently combined diversity streams of user $j$, i.e. $\Phi_{j}$, while the last term contains the magnitude square of the non-coherent combination of the correlated streams of user $i$ and user $j$, i.e. $\Psi$. Recall that the diversity gain is obtained when statistically independent (uncorrelated) streams are used. Hence, only the first term in (10) contributes to the diversity gain. The correlated streams in the last term of (10) is allowed to combine non-coherently as it does not contribute to the diversity gain. Note that the last term does not affect the diversity gain contributed by the first term, as it has zero phase.

The mutual information of user $i$ can be written in similar form as in (7). The received signal and the mutual information of user $j$ can be derived easily. It is worth mentioning that the beamforming structure in (9) reduces the overhead needed to feed back the CSI of the effective scalar channel carrying the desired message of user $i$ and $j$. Specifically, user $i$ and user $j$ only need to know the magnitude of scalar $\left|\mathbf{h}_{i}^{T} \mathbf{F}_{i, j} \mathbf{h}_{j}\right|$, which can be fed back from the relay using a common feedback channel with only half the amount of overhead needed in case I and case II with null-space vector selection. The computational complexity of this coherent combining scheme is at most $\mathcal{O}\left(M N^{3}\right)$ flops, which is no worse than the selection scheme discussed previously.

\section{ERGODIC CAPACITY ANALYSIS}

This section provides the analytical results on the ergodic capacity for case I, where the proposed NC-SM scheme achieves higher multiplexing gain than existing schemes ${ }^{4}$. Since we consider symmetrical channels, i.e. all user nodes

\footnotetext{
${ }^{4}$ The high SNR approximation used in deriving the ergodic capacity in this section is too coarse to capture the power gain (or array gain) due to the use of additional antennas in case II. As a result, we do not include the capacity expression for case II.
}

have the same channel statistics, it is sufficient to study the single-user ergodic capacity. Define the single-user ergodic capacity as the per-user long term data rate a system can support, which can be expressed as

$$
C_{\text {erg }}=\int_{0}^{\infty} \mathcal{I} f_{\mathcal{I}}(\mathcal{I}) d \mathcal{I},
$$

where $f_{\mathcal{I}}(\mathcal{I})$ is the probability density function (PDF) of the mutual information $\mathcal{I}$. Recall the mutual information of the proposed protocol in (7) where Gaussian coding is assumed. Note that $\mathbf{F}_{i, j}=\mathbf{w}_{i, j} \mathbf{w}_{i, j}^{T}$. We drop the subscript of $\mathbf{w}$ for simplicity. First, we look into the properties of the variables in $\mathcal{I}$. Represent matrix $\mathbf{W}=\mathbf{w} \mathbf{w}^{H}$. The matrix $\mathbf{W}$ is a positive semi-definite Hermitian matrix and more importantly, $\mathbf{W}$ carries the idempotent property, such that $\mathbf{W} \mathbf{W}^{H}=\mathbf{W}$. Using the associative property of matrix multiplication, the numerator $\left|\mathbf{h}_{i}^{T} \mathbf{w} \mathbf{w}^{T} \mathbf{h}_{j}\right|^{2}$ in (7) can be split into $\left|\mathbf{h}_{i}^{T} \mathbf{w}\right|^{2}\left|\mathbf{w}^{T} \mathbf{h}_{j}\right|^{2}$. We can write each of the magnitude square as vector multiplication, i.e. $\left|\mathbf{h}_{i}^{T} \mathbf{w}\right|^{2}=\mathbf{h}_{i}^{T} \mathbf{W} \mathbf{h}_{i}^{*}$. Applying the idempotent property of $\mathbf{W}$, we can represent $\mathbf{h}_{i}^{T} \mathbf{W h}_{i}^{*}=\mathbf{h}_{i}^{T} \mathbf{W} \mathbf{W}^{H} \mathbf{h}_{i}^{*}=\left\|\mathbf{h}_{i}^{T} \mathbf{W}\right\|^{2}$. Following similar approach, we can write $\left|\mathbf{w}^{T} \mathbf{h}_{j}\right|^{2}=\left\|\mathbf{W}^{T} \mathbf{h}_{j}\right\|^{2}$, where $\mathbf{W}^{T}=$ $\mathbf{W}^{*} \mathbf{W}^{T}$. Define the auxiliary variables $x=\frac{1}{\sigma^{2}}\left\|\mathbf{h}_{i}^{T} \mathbf{W}\right\|^{2}$ and $y=\frac{1}{\sigma^{2}}\left\|\mathbf{W}^{T} \mathbf{h}_{j}\right\|^{2}$. After some algebraic manipulations, we can rewrite the mutual information in (7) as follows,

$$
\mathcal{I}=\frac{1}{2} \log _{2}\left(1+\frac{x y}{(M+1) x+M y+M}\right) .
$$

Note that $\left\|\mathbf{w} \mathbf{w}^{T} \mathbf{h}_{i}\right\|^{2}=\left\|\mathbf{h}_{i}^{T} \mathbf{W}\right\|^{2}$. Define auxiliary variable $z=\frac{x y}{(M+1) x+M y+M}$, we have the following lemma to quantify the cumulative distribution function (CDF) of variable $z$.

Lemma 1: Assuming i.i.d. quasi-static Rayleigh fading and channel reciprocity, the CDF of auxiliary variable $z$ can be expressed as

$$
\begin{aligned}
F_{z}(z) & =1-2 \sigma^{2} \exp \left(-(2 M+1) \sigma^{2} z\right) \sqrt{M(M+1) z^{2}+M z} \\
& \times K_{1}\left(2 \sigma^{2} \sqrt{M(M+1) z^{2}+M z}\right),
\end{aligned}
$$

where $M$ is the number of user pair and $K_{1}(z)$ is the modified Bessel function of second kind.

Proof: Refer to appendix.

Observe that the CDF of $z$ is expressed in terms of modified Bessel function of second kind, which has no closed form solution. Thus, we proceed to the next lemma to obtain the upper and lower bounds for the CDF of variable $z$ in order to ease the difficulty in the subsequent analytical development of the ergodic capacity.

Lemma 2: Assuming i.i.d. quasi static Rayleigh fading and channel reciprocity, the CDF of auxiliary variable $\mathrm{z}$ can be bounded as

$$
\begin{array}{r}
1-\exp \left(-(2 M+1) \sigma^{2} z\right) \leq F_{z}(z) \leq \\
1-\exp \left(-(2 M+1) \sigma^{2} z-2 \sigma^{2} \sqrt{M(M+1) z^{2}+M z}\right) .
\end{array}
$$

Proof: Refer to appendix.

In order to facilitate the analytical development of the ergodic capacity, we use the following high SNR approximation for the upper bound of the CDF of $z$,

$$
\begin{gathered}
1-\exp \left(-(2 M+1) \sigma^{2} z-2 \sigma^{2} \sqrt{M(M+1) z^{2}+M z}\right) \approx \\
1-\exp \left(-(2 M+1+2 \sqrt{M(M+1)}) \sigma^{2} z\right) .
\end{gathered}
$$


Recall that the mutual information is a function of $z$, such that $\mathcal{I}(z)=\frac{1}{2} \log _{2}(1+z)$. According to section 7.4.9 in [16], the expected value of $\mathcal{I}$ (which is the ergodic capacity) can be derived from the following formula

$$
C_{\text {erg }}=\int_{0}^{\infty} \mathcal{I}(z) f_{z}(z) d z .
$$

The density function $f_{z}(z)$ can be obtained by differentiating $F_{z}(z)$ over $z$, i.e. $f_{z}(z)=\frac{d F_{z}(z)}{d z}$. We have the following theorem for the ergodic capacity of the proposed protocol.

Theorem 1: Assuming i.i.d. quasi-static Rayleigh fading and channel reciprocity, the single-user ergodic capacity of the proposed NC-SM protocol can be upper and lower bounded using high SNR approximation, as follows,

$$
c(2 M+1+2 \sqrt{M(M+1)}) \leq C_{\text {erg }} \leq c(2 M+1),
$$

where the function $c(x)=\frac{1}{2} \exp \left(x \sigma^{2}\right) \log _{2}\left(\frac{1}{x \sigma^{2} \exp (\gamma)}\right)$ and $\gamma$ is the Euler constant.

Proof: Refer to appendix.

Remark 1: For fixed number of user pairs, $M$, we can write the lower bound at high SNR, i.e. $\sigma^{2} \rightarrow 0$, as $C_{\text {erg,lower }} \approx$ $\frac{1}{2} \log _{2}\left(\frac{1}{\sigma^{2}}\right)-\frac{1}{2} \log _{2}((2 M+1+2 \sqrt{M(M+1)}) \exp (\gamma))$. The first term of the RHS is a function of SNR while the second term is a constant independent of the SNR. Therefore, for every $3 \mathrm{~dB}$ increase in SNR, there is a $0.5 \mathrm{bits} / \mathrm{s} / \mathrm{Hz}$ increment on the ergodic capacity. In other words, the achievable per-user multiplexing gain is $\frac{1}{2}$, which is independent of the number of user pairs. The second term captures two effects. First, the power loss due to the null-space projection operation used in the beamformer and second, the equal power sharing among $M$ pairs of users. As the number of user pairs increases, the fixed transmission power at the relay is shared by more users. Hence each user gets a smaller portion of the total power as $M$ increases. This explains why the second term increases logarithmically with $M$. Overall, the single-user ergodic capacity decreases as the number of active user pairs increases, but the individual multiplexing gain obtainable by each user is maintained.

\section{Outage Probability and the Diversity and MultipleXing TradeOFF ANalysis}

In this section, we develop the analytical results on the outage probability and the diversity and multiplexing tradeoff of the proposed NC-SM schemes. Since we are only considering symmetric channels, the analysis of single-user outage performance is adequate. Define the outage probability, $P(I<R)$, as the probability that the mutual information of a user falls below the individual target data rate, $R$. The outage performance of the proposed null-space vector selection scheme is analysed, for two obvious reasons. First, it is the generalisation of the proposed protocol to the case, $N \geq 2 M-1$, which includes both case I and case II. For example, when $N=2 M-1$, the proposed null-space vector selection scheme reverts to the proposed scheme in case I. Second, the proposed null-space vector selection scheme is a lower bound for the proposed coherent combining scheme. This argument is verified in the numerical results section. We have the following theorem to capture the outage probability of the proposed NC-SM schemes.

Theorem 2: Assuming i.i.d. quasi-static Rayleigh fading and channel reciprocity, the single-user outage probability of the proposed NC-SM schemes is

$$
P(\mathcal{I}<R)=\left(1-\exp \left(-(2 M+1) \sigma^{2} \zeta\right) \omega K_{1}(\omega)\right)^{N-2(M-1)},
$$

where $\omega=2 \sigma^{2} \sqrt{M(M+1) \zeta^{2}+M \zeta}$ and $\zeta=2^{2 R}-1$.

Proof: Refer to appendix.

Remark 2: At fixed data rate $R$, the auxiliary variable $w \rightarrow 0$ when $\sigma^{2} \rightarrow 0$. Hence, we can use the approximation for the exponent, i.e. $\exp (-x)=1-x$ and also the approximation for the modified $\stackrel{x \rightarrow 0}{B}$ essel function of second kind, i.e. $K_{1}(x)=\frac{1}{x}$, to obtain the high SNR approximation of the outage probability as follows

$$
P(\mathcal{I}<R) \approx\left((2 M+1) \sigma^{2} \zeta\right)^{N-2(M-1)} .
$$

Representing the mean SNR as $\gamma_{0}=\frac{1}{\sigma^{2}}$, it is readily shown that the outage probability decays as $\frac{1}{\gamma_{0} N-2(M-1)}$. The exponent of the SNR defines the rate of decay and it is wellknown as the diversity gain. When the diversity gain is higher, the outage probability decays faster with increasing SNR. The diversity gain achieved by the proposed NC-SM schemes is $N-2 M+2$. It is better than the conventional multi-user zero-forcing scheme. Since the analytical outage probability of the zero-forcing scheme in [6] is not available, we have to make a comparison with the conventional zero-forcing receiver [17] used in MIMO channels with $2 M$ transmitter antennas and $N$ receiver antennas. Provided that $N \geq 2 M$, the diversity gain achieved by each substream in zero-forcing receiver is $N-2 M+1$. Clearly, the proposed NC-SM schemes deliver additional diversity gain as compared to the MIMO zero-forcing receiver, thanks to the beamformer design which only removes the co-channel interference caused by other user pairs. In comparison with the multiple-input and single-output (MISO) upper bound which has optimal diversity gain of $N$, the loss of diversity gain experienced by the proposed scheme due to the block-diagonalisation is $2 M-2$. This is lower than the loss of $2 M-1$ suffered by conventional zero-forcing scheme.

Similar to the MIMO channels, there is a fundamental tradeoff between the rate and reliability in the multi-pair, bidirectional relay channels. In order to quantify this relationship, we derive the diversity and multiplexing tradeoff for the proposed NC-SM schemes. Recall that the diversity and multiplexing gain can be defined as [18]

$$
d \triangleq-\lim _{\gamma_{0} \rightarrow \infty} \frac{\log \left[P_{e}\left(\gamma_{0}\right)\right]}{\log \gamma_{0}} \text { and } r \triangleq \lim _{\gamma_{0} \rightarrow \infty} \frac{R\left(\gamma_{0}\right)}{\log \gamma_{0}},
$$

where $P_{e}$ is the maximum likelihood (ML) probability of detection error, $R$ is the target data rate in bits/Hz/s, $\gamma_{0}$ is the mean SNR and in our case $\gamma_{0}=\frac{1}{\sigma^{2}}$. The outage probability is used to obtain the diversity and multiplexing tradeoff because the ML error probability is tightly bounded by the outage probability at high SNR. Using the result from theorem 2, we obtain the following corollary which quantifies the diversity and multiplexing tradeoff of the proposed NC-SM schemes. 
Corollary 1: The achievable per-user diversity and multiplexing tradeoff of the proposed NC-SM schemes is

$$
d(r)=(1-2 r)(N-2 M+2), \quad 0 \leq r \leq 1,
$$

provided that $N \geq 2 M-1$.

Proof: Refer to appendix.

Remark 3: The maximum diversity gain (when $r=0$ ), describes how fast the outage probability decays with the SNR at fixed data rate. This maximum diversity gain of $N-2 M+2$ is in line with theorem 2 . On the other extreme, the maximum multiplexing gain (when $d=0$ ), describes how the data rate grows with the SNR at fixed outage probability. The multiplexing gain of $1 / 2$, achievable by each user is independent of the number of user pairs, $M$. This agrees with the result in theorem 1 . The multiplexing gain of $1 / 2$ is indeed the best possible gain that can be achieved in the half duplex, two-way relay channels being studied. The total multiplexing gain achievable in the network is the sum of all individual multiplexing gain, which is equal to $M$. Both the individual and network multiplexing gains of the proposed NC-SM schemes outperform the existing schemes in [6] (when $N<2 M$ ) and [7], which are designed for single-pair and extended to multi-pair using time sharing between pairs. The per-user multiplexing gain achievable by comparable schemes [6], [7] with time sharing between pairs is $1 / 2 M$, which decreases as $M$ gets larger. The detailed comparisons are shown in the next section. Simultaneous increase of the data rate (positive $r$ ) and the reliability (positive $d$ ) is possible in the proposed NC-SM schemes, as long as the diversity and multiplexing tradeoff in (21) is satisfied. The proposed NCSM schemes achieve better diversity and multiplexing tradeoff than the zero-forcing scheme (when $N \geq 2 M$ ) for all data rates. Furthermore, the proposed NC-SM schemes offer better tradeoff at high data rate region as compared to the diversity gain optimal scheme, the maximal ratio reception-transmission (MRR-MRT) scheme [7]. These arguments are justified ${ }^{5}$ by the simulation results in the next section.

\section{NumericAl RESUlts}

We present several Monte Carlo simulation results on the single-user ergodic capacity and single-user outage probability, in order to validate the analytical results and assess the performance of the proposed NC-SM schemes in comparison with existing schemes. All schemes assume equal power allocation to enable fair comparisons.

Fig. 2 shows the single-user ergodic capacity versus SNR of the proposed NC-SM scheme for case I, i.e. $N=2 M-1$. The curve generated using Monte Carlo simulation is compared with the analytical bounds in theorem 1 . The number of user pairs is fixed at $M=2$ while the number of antennas at the relay is fixed at $N=3$. The analytical upper and lower bounds approximate the ergodic capacity very well, starting from medium to high SNR, i.e. SNR $>15 \mathrm{~dB}$. Recall that the slope of the ergodic capacity curve characterises the multiplexing gain.

\footnotetext{
${ }^{5}$ Note that the analytical results on the outage probability and the diversity and multiplexing tradeoff of the existing schemes are not available in the literature, which prevent direct analytical comparison. Until this point, the optimal diversity and multiplexing tradeoff for the multi-pair, two-way relay channels with half duplex constraint, remains an open problem.
}

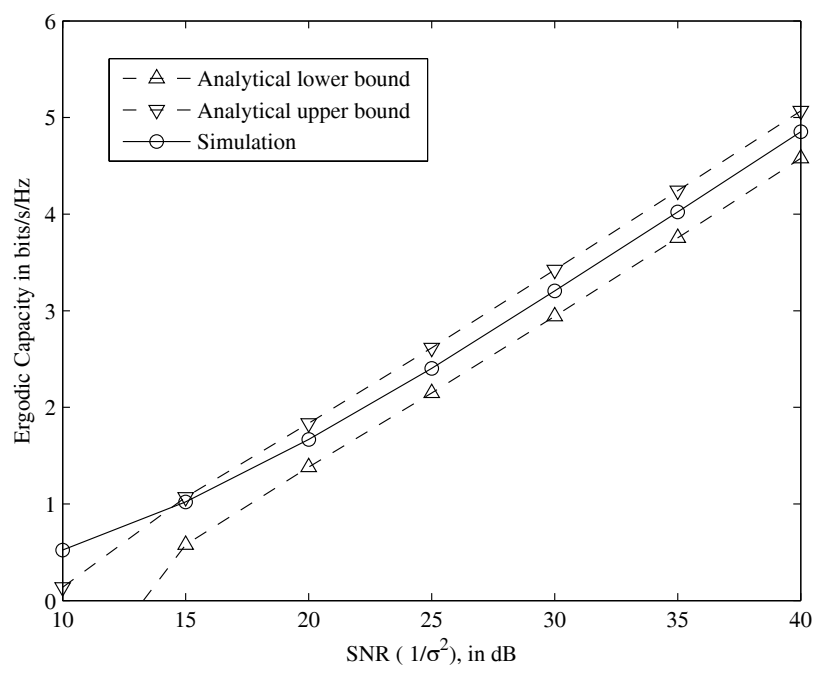

Fig. 2. Single-user ergodic capacity versus SNR of the proposed protocol in comparison with the capacity bounds. Fixed parameters : $M=2$ and $N=3$.

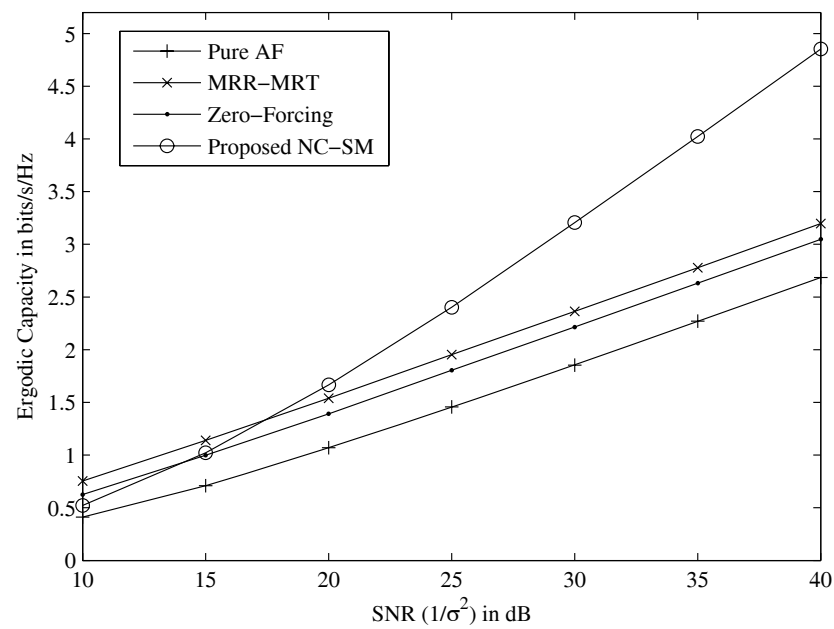

Fig. 3. Single-user ergodic capacity versus SNR of various schemes when $M=2$ and $N=3$.

The simulation result agrees with the analytical bounds that the proposed NC-SM scheme can achieve per-user multiplexing gain of $1 / 2$ (or a total multiplexing gain of $M$ ).

Fig. 3 compares the single-user ergodic capacity versus SNR of the proposed NC-SM scheme and three comparable AF based schemes for case I, i.e. $N=2 M-1$. The fixed parameters are $M=2$ and $N=3$. The baseline schemes: 1. pure $\mathrm{AF}$ (where the relay only forwards power normalised mixture without beamforming); 2. MRR-MRT [7], which is shown to achieve near optimal sum rate in single-pair scenario; and 3. zero-forcing [6]. The baseline schemes are extended to multi-pair using time sharing between pairs. Note that the zero-forcing [6] cannot support all user pairs simultaneously because the zero-forcing criterion requires $N \geq 2 M$. It can be observed that from medium to high SNR, i.e. SNR $>17 \mathrm{~dB}$, the ergodic capacity of the proposed NC-SM scheme outperforms all existing AF based schemes. At high SNR, i.e. SNR=30dB, significant capacity gains of $36 \%, 45 \%$ and $75 \%$ are obtained by the proposed NC-SM scheme in comparison with the MRR- 


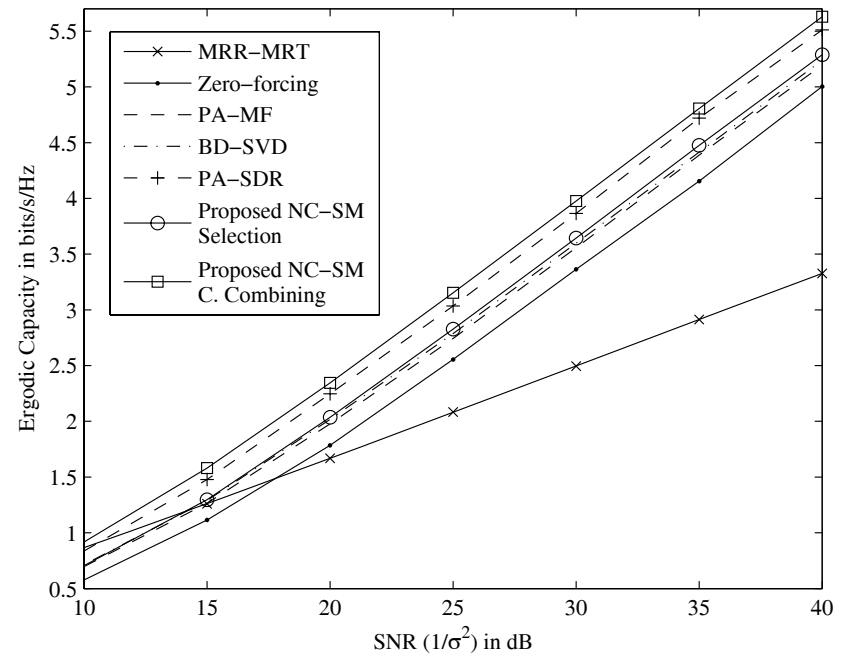

Fig. 4. Single-user ergodic capacity versus SNR of various scheme when $M=2$ and $N=4$.

MRT, zero-forcing, and the pure AF schemes respectively. The proposed protocol demonstrates that spatial multiplexing across different pairs coupled with network coding within same pair is spectrally more efficient. By observing the slope of the curves, it is obvious that the proposed NCSM scheme delivers the highest multiplexing gain among all schemes, thanks to the efficient use of the available degreesof-freedom to spatially support multiple user pairs. At low SNR, system supporting only single-stream, i.e. MRR-MRT, performs better than spatial multiplexing system such as the proposed scheme. Recall that at low SNR, allocating all of the transmission power to the best subchannel (corresponds to single-stream system) is better than distributing the power among all subchannels (corresponds to spatial-multiplexing system).

Fig. 4 shows the single-user ergodic capacity versus SNR of the proposed NC-SM schemes in comparison with the existing AF based schemes, for case II, i.e. $N \geq 2 M$. The fixed parameters are $M=2$ and $N=4$. Recall that in case II, the proposed NC-SM beamformer uses either the null-space vector selection or coherent combining of null-space vectors. The comparable schemes are MRR-MRT, zero-forcing (see [13] for the extension to multi-pair scenario), BD-SVD [13], PAMF and pair-aware with semi-definite relaxation (PA-SDR) [12]. All comparable schemes are able to support all user pairs simultaneously (spatial multiplexing), except for the MRRMRT which uses time-sharing between pairs. From fig. 4, it can be observed that all schemes supporting spatial multiplexing achieve higher ergodic capacity and higher multiplexing gain if compared with the scheme based on time sharing between pairs (MRR-MRT scheme). All spatial multiplexing schemes achieve the same multiplexing gain, evident from the slope of ergodic capacity curves. It can be observed that block-diagonalisation based schemes (including proposed NCSM schemes, BD-SVD, PA-MF and PA-SDR) are able to achieve higher ergodic capacity for any fixed SNR, or deliver power gain for any fixed ergodic capacity, if compared with the zero-forcing scheme. Among all block-diagonalisation

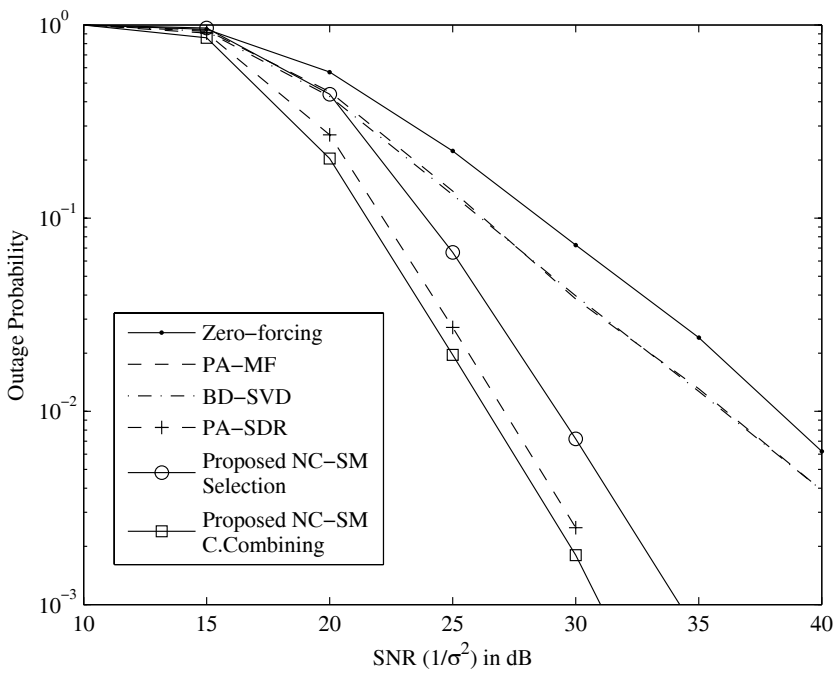

Fig. 5. Single-user outage probability versus SNR when $M=2, N=4$ and $R=2$ bits $/ \mathrm{s} / \mathrm{Hz}$.

based schemes, the proposed NC-SM with coherent combining delivers the best performance. The PA-SDR scheme performs close to the proposed NC-SM with coherent combining, but it comes at the cost of higher computational complexity. PA-SDR has the worst case complexity of $\mathcal{O}\left(N^{7}\right)$ flops as compared to the proposed schemes with at most $\mathcal{O}\left(M N^{3}\right)$ flops. The higher complexity of PA-SDR is due to the use of semi-definite programming in computing the multicast vectors [19]. The NC-SM with null-space vector selection, is about 2 $\mathrm{dB}$ away from the coherent combining scheme. Notice that the PA-MF and BD-SVD schemes do not perform better than the proposed null-space vector selection scheme. Similar to fig.3, MRR-MRT dominates at low SNR.

Besides providing ergodic capacity improvement, blockdiagonalisation offers higher diversity gain as compared to zero-forcing scheme. The diversity gain achieved by the proposed NC-SM schemes can be verified from the outage probability versus SNR curves shown in fig. 5. The fixed parameters are $M=2, N=4$, and $R=2$ bits/s/Hz. Generally, the proposed NC-SM scheme with coherent combining achieves the lowest outage probability. The PA-SDR scheme performs close to the proposed NC-SM with coherent combining while the NC-SM with selection is about $2.5 \mathrm{~dB}$ away from the best scheme. Recall that the slope of the outage probability curve characterises the diversity gain. The proposed NC-SM schemes (both selection and coherent combining schemes) and the PA-SDR are able to achieve a higher diversity gain as compared to the BD-SVD, PA-MF and zero-forcing schemes. The BD-SVD and PA-MF schemes are not able to extract the additional diversity gain offered by block-diagonalisation, due to the non-coherent combining of the diversity streams. Although the PA-SDR scheme is able to extract all the diversity gain, it suffers from higher computational complexity. The proposed NC-SM schemes have lower complexity while being able to achieve all the diversity gain offered by blockdiagonalisation.

The next simulation reveals the tradeoff between the rate and the reliability between different schemes. The curves for 


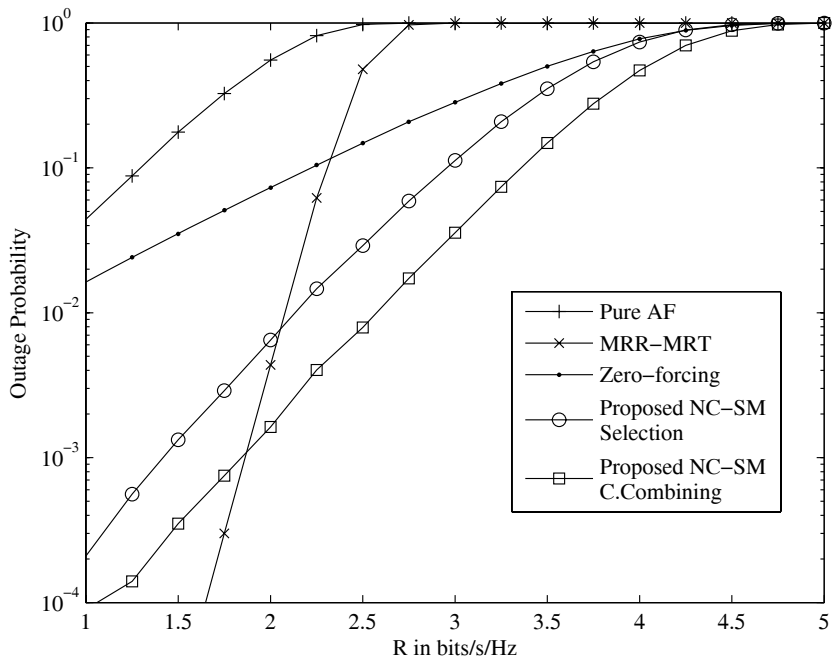

Fig. 6. Single-user outage probability versus target data rate $R$ for various schemes when $M=2, N=4$ and $\mathrm{SNR}=30 \mathrm{~dB}$.

the outage probability versus target data rate, $R$, are shown in fig. 6. The fixed parameters are $M=2, N=4$ and $\mathrm{SNR}=30 \mathrm{~dB}$. The pure AF and MRR-MRT schemes use time sharing between pairs while the zero-forcing and the proposed schemes employ spatial multiplexing. Both the proposed NCSM with selection and the proposed NC-SM with coherent combining achieve lower outage probability if compared with the zero-forcing and the pure AF schemes, at all target data rate. The proposed NC-SM with selection performs close to the proposed NC-SM with coherent combining. Although the zero-forcing scheme also supports spatial multiplexing, it still underperforms the proposed schemes. This is because the zeroforcing scheme has lower diversity gain as compared to the proposed schemes. In general, the proposed schemes have better diversity and multiplexing tradeoff if compared with existing zero-forcing scheme and pure AF scheme. Besides that, the proposed schemes outperform the MRR-MRT at high data rate region, i.e. $R>2 \mathrm{bits} / \mathrm{s} / \mathrm{Hz}$. As an example, at fixed data rate, i.e. $R=3 \mathrm{bits} / \mathrm{s} / \mathrm{Hz}$, the proposed NC-SM with coherent combining is able to maintain $4 \%$ outage while the MRR-MRT scheme completely fails. Although the MRRMRT scheme has the highest diversity gain as compared to all other schemes, it suffers from poor outage performance at high data rate region. In fact, its per-user multiplexing gain is only $1 / 2 M$ due to the use of time sharing between pairs. From implementation perspective, having a system that is able to support a higher data rate at reasonable outage probability is certainly more preferable than a system that delivers lower outage probability but operates at a very low data rate. Along this line, the proposed NC-SM schemes are desirable since they offer better diversity and multiplexing tradeoff at high data rate region. Although not included in the figure, it can be easily verified that the PA-SDR has similar tradeoff performance as the proposed NC-SM schemes, while PA-MF and BD-SVD have similar tradeoff performance as the zero-forcing scheme.

\section{CONCLUSIONS}

The proposed protocol combines analogue network coding and spatial multiplexing, which allows both the relay and the users to participate in interference cancellation. The proposed beamforming schemes yield significant improvement in terms of ergodic capacity and outage probability. The developed analytical bounds approximate the ergodic capacity very well and show that the proposed beamforming scheme achieves higher multiplexing gain than existing schemes. The analytical result on the outage probability quantifies the diversity gain and proves that the proposed beamforming schemes are able to extract all additional diversity gain offered by blockdiagonalisation as compared to zero-forcing. The derived diversity and multiplexing tradeoff reveals the superior rate and reliability tradeoff offered by the proposed schemes. Simulation results agree with the analytical results that the proposed beamforming schemes achieve higher ergodic capacity, lower outage probability and better diversity and multiplexing tradeoff than comparable schemes.

\section{APPENDIX}

Proof of Lemma 1: First, we determine the distribution of the auxiliary variable $x$. With the knowledge of $\mathbf{h}_{i}^{T} \sim \mathcal{C N}\left(0, \mathbf{I}_{N}\right)$ and applying the idempotent property of $\mathbf{W}$, the quadratic form $\mathbf{h}_{i}^{T} \mathbf{W} \mathbf{h}_{i}^{*}$ is shown in [20] to have chi-square distribution with $2 \times \operatorname{trace}(\mathbf{W})$ degrees of freedom. In our case, $\operatorname{trace}(\mathbf{W})=1$. Denote $u=\mathbf{h}_{i}^{T} \mathbf{W h}_{i}^{*}$, the PDF of $u$ reduces to $f_{u}(u)=\exp (-u)$, which is an exponential distribution. Applying change of variables, $u=\sigma^{2} x$, the PDF of variable $x$ can be expressed as $f_{x}(x)=\sigma^{2} \exp \left(-\sigma^{2} x\right)$. Using similar approach, we obtain the PDF of the variable $y$ as $f_{y}(y)=\sigma^{2} \exp \left(-\sigma^{2} y\right)$. Next, we derive the distribution of variable $z=\frac{x y}{(M+1) x+M y+M}$. Knowing that $x$ and $y$ are independently distributed, the cumulative distribution function of $z$ can be written as

$$
F_{z}(z)=\iint_{\frac{x y}{(M+1) x+M y+M}} f_{x}(x) f_{y}(y) d x d y .
$$

Since we know that $x \geq 0$ and $y \geq 0$, we can express $F_{z}(z)$ with the following equation,

$$
\begin{aligned}
F_{z}(z) & =P(0 \leq y \leq(M+1) z, 0 \leq x \leq \infty) \\
& +P\left((M+1) z \leq y \leq \infty, 0 \leq x \leq \frac{M y z+M z}{y-(M+1) z}\right),
\end{aligned}
$$

which is then written in integral form as follows

$$
\begin{aligned}
F_{z}(z) & =\int_{0}^{(M+1) z} \sigma^{2} \exp \left(-\sigma^{2} y\right) \int_{0}^{\infty} \sigma^{2} \exp \left(-\sigma^{2} x\right) d x d y \\
& +\int_{(M+1) z}^{\infty} \sigma^{2} \exp \left(-\sigma^{2} y\right) \\
& \times \int_{0}^{\frac{M y z+M z}{y-(M+1) z}} \sigma^{2} \exp \left(-\sigma^{2} x\right) d x d y .
\end{aligned}
$$

The first pair of the integrals in (24) can be calculated easily,

$$
\begin{array}{r}
\int_{0}^{(M+1) z} \sigma^{2} \exp \left(-\sigma^{2} y\right) \int_{0}^{\infty} \sigma^{2} \exp \left(-\sigma^{2} x\right) d x d y= \\
1-\exp \left(-(M+1) \sigma^{2} z\right),
\end{array}
$$


while the second pair of integrals in (24) is

$$
\begin{aligned}
& \int_{(M+1) z}^{\infty} \sigma^{2} \exp \left(-\sigma^{2} y\right) \int_{0}^{\frac{M y z+M z}{y-(M+1) z}} \sigma^{2} \exp \left(-\sigma^{2} x\right) d x d y \\
& =\int_{(M+1) z}^{\infty} \sigma^{2} \exp \left(-\sigma^{2} y\right) \\
& \times\left[1-\exp \left(-\sigma^{2} \frac{M y z+M z}{y-(M+1) z}\right)\right] d y, \\
& =\overline{\overline{(M}} \exp \left(-(M+1) \sigma^{2} z\right) \\
& -\sigma^{2} \exp \left(-(2 M+1) \sigma^{2} z\right) \int_{0}^{\infty} \exp \left(-\alpha u-\frac{\beta}{u}\right) d u,
\end{aligned}
$$

where $\alpha=\sigma^{2}$ and $\beta=\left(M(M+1) z^{2}+M z\right) \sigma^{2}$. There is no closed-form solution available for the integral in (26). However, we can represent the integral according to equation 3.471.9 in [21], which is shown here for convenience,

$$
\int_{0}^{\infty} \exp \left(-\alpha u-\frac{\beta}{u}\right) d u=2 \sqrt{\frac{\beta}{\alpha}} K_{1}(2 \sqrt{\alpha \beta})
$$

where $K_{1}(z)$ is the modified Bessel function of second kind. Substituting (27) into (26), we have the following

$$
\begin{aligned}
& \int_{(M+1) z}^{\infty} \sigma^{2} \exp \left(-\sigma^{2} y\right) \int_{0}^{\frac{M y z+M z}{y(M+1) z}} \sigma^{2} \exp \left(-\sigma^{2} x\right) d x d y \\
& =\exp \left(-(M+1) \sigma^{2} z\right)-2 \sigma^{2} \exp \left(-(2 M+1) \sigma^{2} z\right) \\
& \times \sqrt{M(M+1) z^{2}+M z} K_{1}\left(2 \sigma^{2} \sqrt{M(M+1) z^{2}+M z}\right) .
\end{aligned}
$$

Combining (25) and (28), the CDF of $z$ can be obtained and the lemma is proved.

Proof of Lemma 2: Since the CDF of $z$ is related to the Bessel function, it can be bounded by first determining the upper and lower bounds for the modified Bessel function of second kind. From [22], the modified Bessel function of second kind can be bounded as $\frac{\exp (-x)}{x} \leq K_{1}(x) \leq \frac{1}{x}$. Using the bounded $K_{1}(x)$, the upper and lower bounds for the CDF of $z$ can be obtained and thus the lemma is proved.

Proof of Theorem 1: Since the distribution function $F_{z}(z)$ is bounded, the density function $f_{z}(z)$ is bounded as well. It is shown in [22] that the inequalities between the expected value of two exponential density functions of $x$ is such $\int_{0}^{\infty} x f_{1}(x) d x<\int_{0}^{\infty} x f_{2}(x) d y$ happens when $F_{1}(x) \geq F_{2}(x)$, and $\int_{0}^{\infty} x f_{1}(x) d x>\int_{0}^{\infty} x f_{2}(x) d y$ happens when $F_{1}(x) \leq F_{2}(x)$. Using this relationship, and let the function $g(u)=\int_{0}^{\infty} \frac{1}{2} u \exp (-u z) \log _{2}(1+z) d z$, we can have the following bounds for the ergodic capacity,

$g\left((2 M+1) \sigma^{2}+2 \sqrt{M(M+1)} \sigma^{2}\right) \leq C_{\text {erg }} \leq g\left((2 M+1) \sigma^{2}\right)$

We solve the function $g(u)$ as follows

$$
\begin{aligned}
g(u) & =\int_{0}^{\infty} \frac{1}{2} u \exp (-u z) \log _{2}(1+z) d z \\
& =\frac{1}{2} u \log _{2}(e) \int_{0}^{\infty} \exp (-u z) \ln (1+z) d z \\
& =\frac{1}{(a)} \log _{2}(e) \exp (u) E_{1}(u),
\end{aligned}
$$

where $E_{1}$ is the exponential integral and the equality (a) is obtained using equation 4.337.2 in [21]. The exponential integral, $E_{1}(u)$ can be represented as series expansion which is found in section 5.1.11 in [23],

$$
E_{1}(u)=-\gamma-\ln u-\sum_{n=1}^{\infty} \frac{(-1)^{n} u^{n}}{n n !},
$$

where $\gamma$ is the Euler constant. When $u \rightarrow 0$, the following approximation can be made

$$
E_{1}(u) \approx-\gamma-\ln u \text { when } u \rightarrow 0 .
$$

At high SNR, the noise power $\sigma^{2} \rightarrow 0$, therefore we can have the following approximation

$$
g(u) \approx \frac{1}{2} \log _{2}(e) \exp (u)(-\gamma-\ln u) .
$$

Substituting (33) into (29) and performing some algebraic manipulations, we obtain the upper and lower bounds for the ergodic capacity and the theorem is proved.

Proof of Theorem 2: Define the variables $x_{k}=$ $\frac{1}{\sigma^{2}}\left\|\mathbf{h}_{i}^{T} \mathbf{W}(k)\right\|^{2}$ and $y_{k}=\frac{1}{\sigma^{2}}\left\|\mathbf{W}(k)^{T} \mathbf{h}_{j}\right\|^{2}$, where $\mathbf{W}(k)=$ $\mathbf{w}(k) \mathbf{w}^{H}(k)$, we can rewrite the beamforming selection criterion in (8) as follows,

$$
\begin{aligned}
\arg \max _{k=1, \ldots, N-2(M-1)} & \frac{1}{2} \log _{2}\left(1+\frac{x_{k} y_{k}}{(M+1) x_{k}+M y_{k}+M}\right) \\
+ & \frac{1}{2} \log _{2}\left(1+\frac{x_{k} y_{k}}{M x_{k}+(M+1) y_{k}+M}\right) .
\end{aligned}
$$

It can be observed that the first term and the second term of the objective function are almost identical, except at the denominator, where the multiplier of variable $x_{k}$ and the multiplier of variable $y_{k}$ interchange. The minor difference between the first term and the second term does not result in any statistical difference. It can be verified that the CDF of the first term and the second term are the same. In order to simplify the analytical development, we reduce the sum rate criterion in (34) into

$$
\arg \max _{k=1, \ldots, N-2(M-1)}\left(\frac{x_{k} y_{k}}{(M+1) x_{k}+M y_{k}+M}\right),
$$

where only the instantaneous SNR of user $i$ is maximised. We name it as the individual rate criterion. Fig. 7 shows the outage probability versus SNR of the proposed null-space vector selection scheme under two different selection criteria in comparison with the baseline zero-forcing scheme. In the simulation, we fixed $N=4, M=2$ and $R=2 \mathrm{bits} / \mathrm{s} / \mathrm{Hz}$. From fig. 7, it can be easily seen that the outage probability obtained using sum rate criterion is almost the same as the outage probability obtained using individual rate criterion. This verifies that the individual rate criterion is a very accurate approximation for the sum rate criterion.

Using the simplified selection criterion in (35), we can arrange the mutual information of a user, in ascending order as follows,

$$
\mathcal{I}_{(1)} \leq \mathcal{I}_{(2)} \leq \ldots \leq \mathcal{I}_{(N-2(M-1))}
$$

The null-space vector producing the maximum mutual information, $\mathcal{I}_{(N-2(M-1))}$ is selected. When the $k$ th null-space 


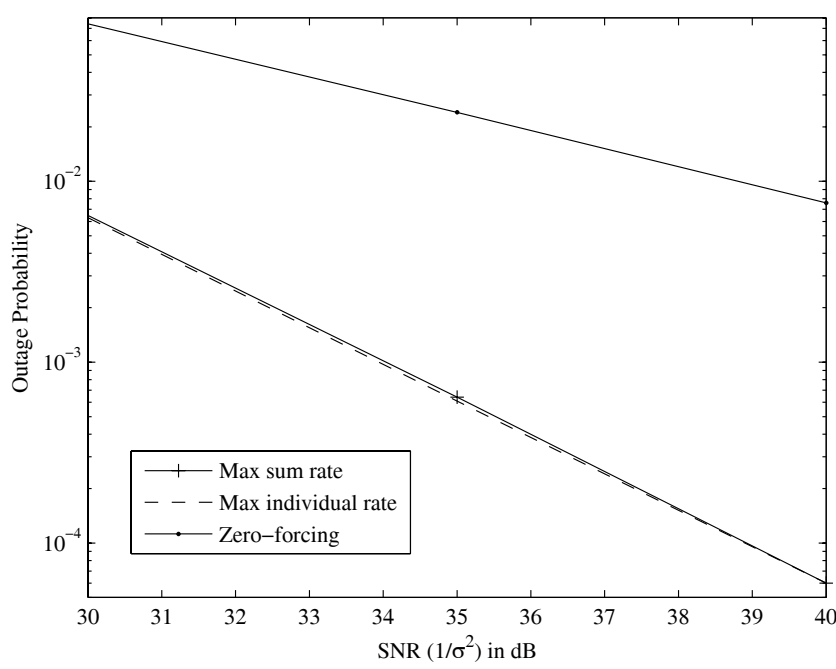

Fig. 7. Outage probability versus SNR under 2 different selection criteria in comparison with the baseline zero-forcing scheme. The fixed parameters are $M=2, N=4$ and $R=2 \mathrm{bits} / \mathrm{s} / \mathrm{Hz}$.

vector is randomly chosen, the outage probability can be described as

$$
P(\mathcal{I}(k)<R)=P\left(\frac{x_{k} y_{k}}{(M+1) x_{k}+M y_{k}+M}<2^{2 R}-1\right) .
$$

Introduce the auxiliary variable $z_{k}=\frac{x_{k} y_{k}}{(M+1) x_{k}+M y_{k}+M}$. From the result in lemma 1 , we can easily obtain the CDF of $z_{k}$ as

$$
P\left(z_{k}<R\right)=1-\exp \left(-(2 M+1) \sigma^{2} \zeta\right) \omega K_{1}(\omega),
$$

where $\omega=2 \sigma^{2} \sqrt{M(M+1) \zeta^{2}+M \zeta}$ and $\zeta=2^{2 R}-1$. In order to determine the outage probability when the best null-space vector is selected, order statistics [24] is used. Specifically, the outage probability is

$$
P(\mathcal{I}<R)=\left(P\left(z_{k}<R\right)\right)^{N-2(M-1)},
$$

where $\mathcal{I}=\mathcal{I}_{(N-2(M-1))}$ is the mutual information when the best null-space vector is used. Substituting (38) into (39), the theorem is proved.

Proof of Corollary 1: As shown in remark 2 of theorem 2, the high SNR approximation for the outage probability can be written as

$$
P(\mathcal{I}<R) \approx\left((2 M+1) \sigma^{2} \zeta\right)^{N-2(M-1)} .
$$

Substituting $R=r \log _{2}\left(\gamma_{0}\right)$ into (40), we have

$$
\begin{aligned}
P(\mathcal{I}<R) & \approx\left((2 M+1)\left(\frac{1}{\gamma_{0}}\right)\left(\gamma_{0}^{2 r}-1\right)\right)^{N-2(M-1)} \\
& \doteq \gamma_{0}^{-(1-2 r)(N-2(M-1))}
\end{aligned}
$$

where we use special symbol $\doteq$ to denote the exponential equality, i.e. $f\left(\sigma^{2}\right) \doteq \gamma_{0}^{n}$ to denote $\lim _{\gamma_{0} \rightarrow \infty} \frac{\log _{2} f\left(\gamma_{0}\right)}{\log _{2} \gamma_{0}}=n$. From the exponential equality, the diversity and multiplexing tradeoff of the proposed NC-SM scheme is readily shown as

$$
d(r)=(1-2 r)(N-2 M+2),
$$

\section{REFERENCES}

[1] R. Ahlswede, N. Cai, S.-Y. R. Li, and R. W. Yeung, "Network information flow," IEEE Trans. Inf. Theory, vol. 46, no. 4, pp. 1204-1216, 2000.

[2] R. F. Wyrembelski, T. J. Oechtering, and H. Boche, "Decode-andforward strategies for bidirectional relaying," in Proc. IEEE PIMRC '08, Sep. 2008, pp. 1-6.

[3] S. Katti, S. Gollakota, and D. Katabi, "Embracing wireless interference: analog network coding," in Proc. SIGCOMM '07, pp. 397-408.

[4] S. Zhang, S. Liew, and P. Lam, "Hot topic: physical layer network coding," in Proc. ACM MobiCom '06, pp. 358-365.

[5] T. J. Oechtering and H. Boche, "Optimal transmit strategies in multiantenna bidirectional relaying," in Proc. IEEE ICASSP 2007, vol. 3, Apr. 2007, pp. III-145-III-148.

[6] T. Unger and A. Klein, "On the performance of two-way relaying with multiple-antenna relay stations," in Proc. 16th IST Mobile Wireless Commun. Summit, July 2007, pp. 1-5.

[7] Y.-C. Liang and R. Zhang, "Optimal analogue relaying with multiantennas for physical layer network coding," in Proc. IEEE ICC '08, May 2008, pp. 3893-3897.

[8] N. Lee, H. J. Yang, and J. Chun, "Achievable sum-rate maximizing af relay beamforming scheme in two-way relay channels," in Proc. IEEE ICC Workshops '08, May 2008, pp. 300-305.

[9] M. Chen and A. Yener, "Multiuser two-way relaying for interference limited systems," in IEEE ICC '08, May 2008.

[10] C. Esli and A. Wittneben, "One- and two-way decode-and-forward relaying for wireless multiuser mimo networks," in Proc. IEEE GLOBECOM 2008, Nov. 2008, pp. 1-6.

[11] Q. H. Spencer, A. L. Swindlehurst, and M. Haardt, "Zero-forcing methods for downlink spatial multiplexing in multiuser mimo channels," IEEE Trans. Signal Process., vol. 52, no. 2, pp. 461-471, Feb. 2004.

[12] A. U. T. Amah and A. Klein, "Pair-aware transceive beamforming for non-regenerative multi-user two-way relaying," in Proc. IEEE ICASSP 2010, Mar. 2010, pp. 2506-2509.

[13] E. Yilmaz, R. Zakhour, D. Gesbert, and R. Knopp, "Multi-pair two-way relay channel with multiple antenna relay station," in IEEE ICC 2010, May 2010.

[14] C. Y. Leow, Z. Ding, K. K. Leung, D. L. Goeckel, and D. Towsley, "Analogue network coding for multi-pair, bidirectional relay channels," in Sensor Signal Process. Defence (SSPD 2010).

[15] E. Biglieri, R. Calderbank, A. Constantinides, A. Goldsmith, A. Paulraj, and H. V. Poor, MIMO Wireless Communications. Cambridge University Press, 2007.

[16] D. Stirzaker, Elementary Probability, 2nd edition. Cambridge University Press, 2003.

[17] D. Gore, R. W. Heath, Jr., and A. Paulraj, "Transmit selection in spatial multiplexing systems," IEEE Commun. Lett., vol. 6, no. 11, pp. 491-493, Nov. 2002.

[18] L. Zheng and D. Tse, "Diversity and multiplexing: a fundamental tradeoff in multiple-antenna channels," IEEE Trans. Inf. Theory, vol. 49, no. 5, pp. 1073-1096, May 2003.

[19] N. D. Sidiropoulos, T. N. Davidson, and Z.-Q. Luo, "Transmit beamforming for physical-layer multicasting," IEEE Trans. Signal Process., vol. 54, no. 6, pp. 2239-2251, June 2006.

[20] R. J. Muirhead, Aspects of Multivariate Statistical Theory, ser. Wiley Series in Probability and Mathematical Statistics. John Wiley \& Sons, 1982.

[21] I. S. Gradshteyn and I. M. Ryzhik, Table of Integrals, Series and Products, 6th edition, A. Jeffrey and D. Zwillinger, editors. Academic Press, 2000.

[22] Z. Ding, K. K. Leung, D. L. Goeckel, and D. Towsley, "On the study of network coding with diversity," IEEE Trans. Wireless Commun., vol. 8, no. 3, pp. 1247-1259, Mar. 2009.

[23] M. Abramowitz and I. A. Stegun, Handbook of Mathematical Functions with Formulas, Graphs, and Mathematical Tables, 9th edition. Dover, 1970.

[24] H. A. David and H. N. Nagaraja, Order Statistics, 3rd edition. Wiley Interscience, 2003.

and the corollary is proved. 


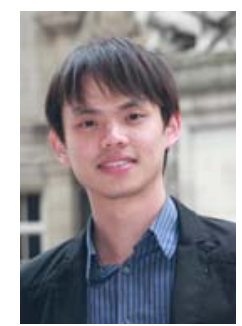

Chee Yen Leow obtained his B.Eng. degree in Computer Engineering from Universiti Teknologi Malaysia in 2007. Since July 2007, he has been an academic staff in the Faculty of Electrical Engineering, Universiti Teknologi Malaysia. Currently, he is on his study leave to pursue Ph.D. degree in Electrical Engineering at Imperial College London, under the supervision of Prof. Kin K. Leung. His research interests include cooperative and relay communications in multi-user networks, linear precodings, communication theory and convex optimisation.

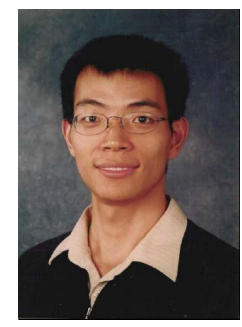

Zhiguo Ding ( $\mathrm{S}^{\prime}$ 03-M'05) received his B.Eng. in Electrical Engineering from the Beijing University of Posts and Telecommunications in 2000, and the $\mathrm{Ph} . \mathrm{D}$. degree in Electrical Engineering from Imperial College London in 2005.From July 2005 to June 2010, he was working in Queen's University Belfast, Imperial College and Lancaster University. Since July 2010, he has been with Newcastle University as a Lecturer. His research interests are cross-layer optimization, cooperative diversity, statistical signal processing and information theory.

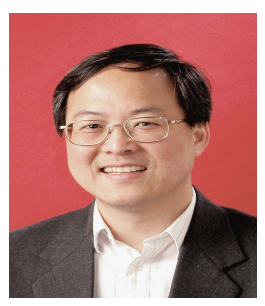

Kin K. Leung received his B.S. degree (with firstclass honors) from the Chinese University of Hong Kong in 1980, and his M.S. and Ph.D. degrees in computer science from University of California, Los Angeles, in 1982 and 1985, respectively.

He started his career at AT\&T Bell Labs in 1986 and worked at its successor companies, AT\&T Labs and Bell Labs of Lucent Technologies, until 2004. Since then, he has been the Tanaka Chair Professor in Internet Technology at Imperial College in London. His research interests include network resource allocation, MAC protocol, TCP/IP protocol, distributed optimization algorithms, mobility management, network architecture, real-time applications and teletraffic issues for broadband wireless networks, wireless sensor and adhoc networks. He is also interested in a wide variety of wireless technologies, including IEEE 802.11, 802.16, and 3G and future generation cellular networks.

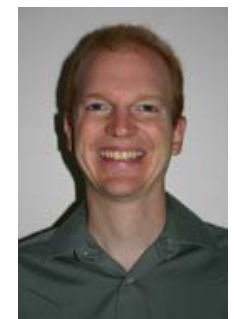

Dennis Goeckel split time between Purdue University and Sundstrand Corporation from 1987-1992, receiving his BSEE from Purdue in 1992. From 1992-1996, he was a National Science Foundation Graduate Fellow and then Rackham Pre-Doctoral Fellow at the University of Michigan, where he received his MSEE in 1993 and his Ph.D. in 1996, both in Electrical Engineering with a specialty in Communication Systems. In September 1996, he joined the Electrical and Computer Engineering department at the University of Massachusetts, where he is currently a Professor. His current research interests are in the areas of communication systems and wireless network theory.

Dr. Goeckel was the recipient of a 1999 CAREER Award from the National Science Foundation for "Coded Modulation for High-Speed Wireless Communications." He was a Lilly Teaching Fellow at UMass-Amherst for the 2000-2001 academic year and received the University of Massachusetts Distinguished Teaching Award in 2007. He served as an Associate Editor for the IEEE JOURNAL ON SELECTEd AREAS IN COMMUNICATIONS: Wireless COMMUNICATION Series during its transition to the IEEE TRANSACTIONS ON WiRELESS COMMUNICATIONS from 1999-2002, an Associate Editor for the IEEE TRANSACTIONS ON COMMUNICATIONS from 2006-2010, a Technical Program Committee Co-Chair for the Communication Theory Symposium at IEEE Globecom 2004, and a Technical Program Committee Co-Chair for the Wireless Communications Symposium at IEEE GlobeCom 2008. He is currently an Associate Editor for the IEEE TRANSACTIONS ON Mobile Computing. 